\title{
Antithrombotic alternatives for stroke prevention in atrial fibrillation: critical differences and remaining questions
}

\author{
James S Kalus
}

Henry Ford Hospital, Detroit, USA

\begin{abstract}
Three therapeutic alternatives for prevention of stroke in patients with atrial fibrillation are available in dabigatran (an oral direct thrombin inhibitor), rivaroxaban, and apixaban (both oral blood coagulation factor Xa inhibitors). Compared with warfarin, these new agents have a more predictable pharmacodynamic response and fewer major clinically relevant drug-drug interactions. These agents also have few, if any, food-drug interactions, and infrequent or no need for routine laboratory monitoring. These agents also bring new disadvantages, particularly lack of clearly defined reversal strategies, inability to effectively monitor patient response, and higher cost. Selection of the most appropriate oral antithrombotic agent for a given patient is dependent on clinician knowledge of the similarities and critical differences between the available antithrombotic medications.

Citation

Provenance

Dates

Copyright

Correspondence address

Email address

Competing interests

Abbreviations

Kalus JS. Antithrombotic alternatives for stroke prevention in atrial fibrillation: critical differences and remaining questions Drugs in Context 2013; 212251. doi: 10.7573/dic.212251

Invited; externally peer reviewed

Submitted: 1 March 2013; Accepted, subject to peer review: 5 March 2013; Published: 16 April 2013

(c) 2013 Kalus JS. This is an open-access article distributed under the terms of the Creative Commons Attribution License (CC-BY-NC-ND 3.0) which allows unrestricted sharing, copying and distribution for personal use provided it is properly attributed. Commercial use is not permitted.

James S Kalus, PharmD, BCPS (AQ CV), Senior Manager, Patient Care Services, Henry Ford Hospital, Detroit, MI 48202, USA

Jkalus1@hfhs.org

James $\mathrm{S}$ Kalus has declared that there are no competing interests.

aPCC, activated prothrombin complex concentrates; aPTT, activated partial thromboplastin time; CHADS2, a scoring system with one point each for congestive heart failure (C), high blood pressure (H), age 75 or older (A), and diabetes (D), and two points for a previous stroke (S2) or transient ischemic attack; CYP450, cytochrome P-450; ECT, ecarin clotting time; INR, international normalized ratio; PGP, p-glycoprotein; PT, prothrombin time; TT, thrombin time

Keywords
\end{abstract}

\section{Introduction}

For the first time since the 1950s, there is an oral antithrombotic agent alternative to warfarin. Three therapeutic alternatives are available in dabigatran (an oral direct thrombin inhibitor), rivaroxaban, and apixaban (both oral blood coagulation factor $\mathrm{Xa}$ inhibitors). The promise of these new agents includes more predictable pharmacodynamic response, fewer major clinically relevant drug-drug interactions, few (if any) food-drug interactions, and infrequent or no need for routine laboratory monitoring. These agents also bring new disadvantages, particularly lack of clearly defined reversal strategies, inability to effectively monitor patient response, and higher cost.

While the above-noted advantages and disadvantages are present with the new oral antithrombotic agents as a group, there are differences between the individual agents. Recent approval of the third oral antithrombotic alternative to warfarin, apixaban, highlights the fact that expertise in selecting the appropriate antithrombotic agent is essential for the effective management

Drugs in Context - The journal of interventions in clinical practice This full text PDF downloaded from www.drugsincontext.com Drugs in Context 2013; 212251. ISSN 1740-4398

Copyright (C 2013 Kalus JS. Distributed under the terms of the Creative Commons Attribution License CC-BY-NC-ND 3.0.

No other uses without permission. of atrial fibrillation. Therefore, in order to select the right oral antithrombotic agent for a given patient, clinicians must have knowledge of the similarities and critical differences between the available antithrombotic medications.

\section{Similarities and critical differences}

\section{Efficacy and safety}

Key characteristics of the clinical trials evaluating the new oral antithrombotic agents are summarized in Table 1. Apixaban is the only agent studied in comparison to aspirin in a patient population unable to take warfarin (AVERROES, Apixaban versus acetylsalicylic acid to prevent stroke in atrial fibrillation patients who have failed or are unsuitable for vitamin $\mathrm{K}$ antagonist treatment) [1]. The RE-LY (Randomized Evaluation of Long-Term Anticoagulation Therapy, dabigatran vs warfarin), ROCKETAF (Rivaroxaban Once Daily Oral Direct Factor Xa Inhibition Compared with Vitamin K Antagonism for Prevention of Stroke and Embolism Trial in Atrial Fibrillation, rivaroxaban vs warfarin), and ARISTOTLE (Apixaban for Reduction in Stroke and Other Thromboembolic Events in Atrial Fibrillation, apixaban $v s$ warfarin) studies were all randomized, non-inferiority studies of approximately similar sample size $(-15,000-18,000)[2-4]$. 
Table 1. Major clinical trials of the new oral antithrombotic agents [1-4]

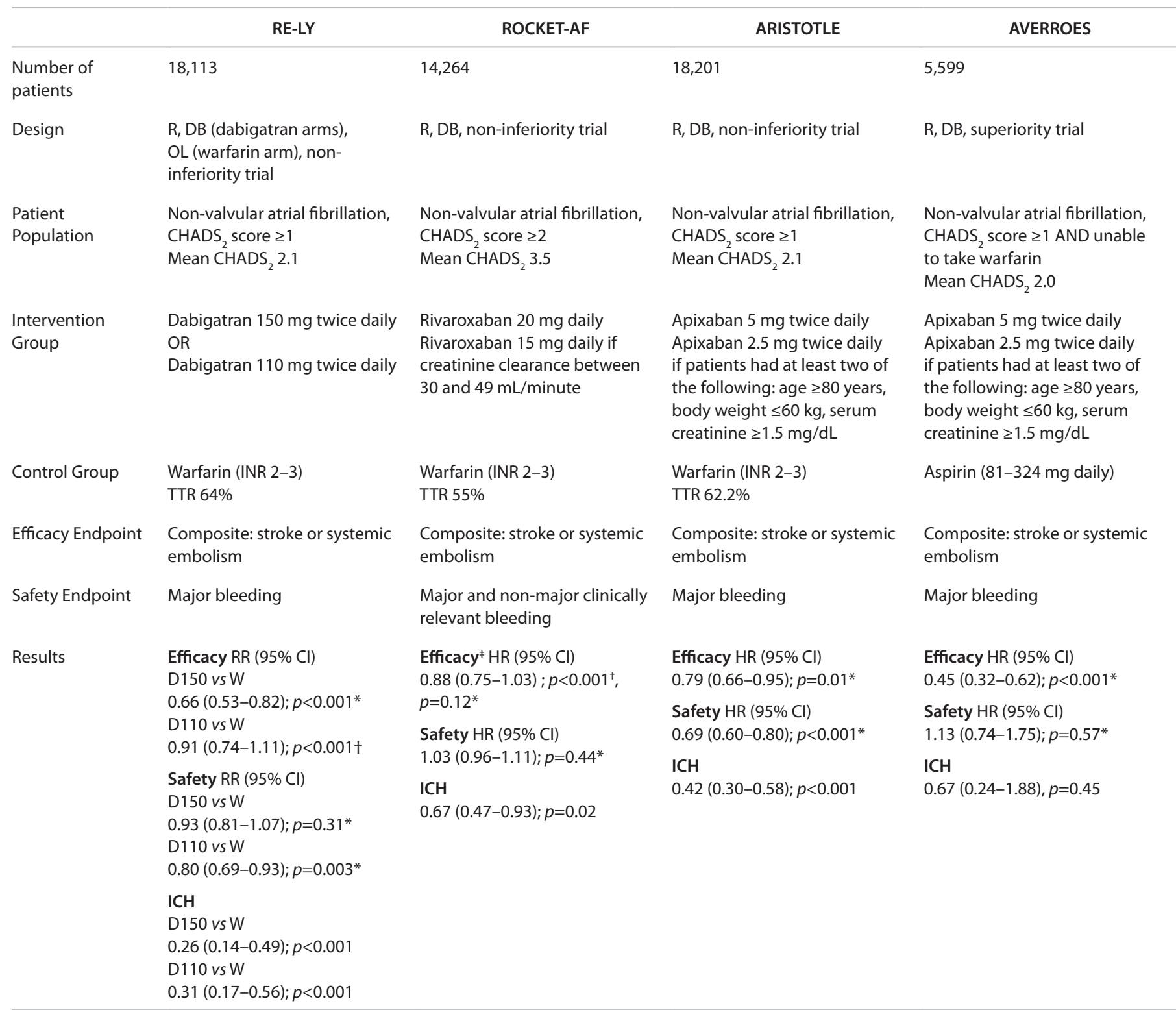

ARISTOTLE, Apixaban for Reduction in Stroke and Other Thromboembolic Events in Atrial Fibrillation, apixaban vs warfarin; AVERROES, Apixaban versus acetylsalicylic acid to prevent stroke in atrial fibrillation patients who have failed or are unsuitable for vitamin K antagonist treatment; RE-LY, Randomized Evaluation of Long-Term Anticoagulation Therapy, dabigatran vs warfarin; ROCKET-AF, Rivaroxaban Once Daily Oral Direct Factor Xa Inhibition Compared with Vitamin K Antagonism for Prevention of Stroke and Embolism Trial in Atrial Fibrillation, rivaroxaban vs warfarin; $95 \% \mathrm{Cl}, 95 \%$ confidence interval; D1 10, dabigatran 110 mg; D150, dabigatran 150 mg; DB, double-blind; HR, hazard ratio; ICH, intracranial hemorrhage; INR, international normalized ratio; OL, open-label; $R$, randomized; $R R$, relative risk; TTR, mean percent of time in the therapeutic range; $W$, warfarin.

${ }^{*} p$-value for superiority; ${ }^{\dagger} p$-value for non-inferiority; ${ }^{*}$ Intention-to-treat results presented doi: 10.7573/dic.212251.t001

A notable difference between the designs of these three studies is that RE-LY was a partially open-label trial in which investigators and study patients knew whether they were receiving dabigatran or warfarin, while the others were double-blinded trials. The non-inferiority margins and efficacy endpoint (composite of stroke and systemic embolism) were the same in all studies; however, the safety endpoint differed slightly. All three studies included major bleeding (International Society on Thrombosis and Hemostasis definition [5]) in the primary safety endpoint; however, the ROCKET-AF study included clinically relevant non-major bleeding in addition to major bleeding as a primary composite safety endpoint.

Inclusion and exclusion criteria were another area of difference between the different trials. All studies included patients with non-valvular atrial fibrillation; however, stroke risk (as defined by the $\mathrm{CHADS}_{2}$ score [6]) differed between studies. The minimum $\mathrm{CHADS}_{2}$ score required for entry into the RE-LY and ARISTOTLE studies was 1 , while the minimum score for entry into ROCKET-AF was 2. Ultimately, this resulted in recruitment of a patient population with a higher stroke risk in ROCKET- 
$\mathrm{AF}$ (median $\mathrm{CHADS}_{2}$ score=3) than in RE-LY (mean CHADS score $=2.1$ ) and ARISTOTOLE (mean $\mathrm{CHADS}_{2}$ score $=2.1$ ).

Management of renal dysfunction also differed between the three studies. In RE-LY, patients were excluded if creatinine clearance was less than $30 \mathrm{~mL} /$ minute [2]. In ROCKET-AF, patients received rivaroxaban $20 \mathrm{mg}$ daily, if the creatinine clearance was greater than $50 \mathrm{~mL} /$ minute and rivaroxaban $15 \mathrm{mg}$ daily, if the creatinine clearance was between 30 and $49 \mathrm{~mL} /$ minute [3]. In the ARISTOTOLE study, a reduced dose of apixaban was given to patients who had two or more of the following: age greater than 80 years, body weight less than $60 \mathrm{~kg}$, or serum creatinine greater than $1.5 \mathrm{mg} / \mathrm{dL}$ [4]. These differences in the way renal dysfunction was handled in the three major studies have implications for interpretation of labeling and renal dosage adjustment, and will be discussed below.

Quality of warfarin management, as measured by the mean time in INR range, was slightly different between the RE-LY (64\%), ROCKET-AF (55\%), and ARISTOTLE (62.2\%) studies. Given the small differences in time in range, and lack of a clear benchmark for defining anticoagulation management quality with this measure, it is difficult to interpret whether the observed differences are clinically meaningful or not. Of note, post hoc analysis of the RE-LY study evaluated the relationship between quality of anticoagulation at a study center and outcome of patients enrolled at that center. This analysis found that dabigatran was effective across all centers, but seemed to be most effective among patients who were managed by study centers where warfarin management quality was of lower quality (i.e. lower time in range) [7]. This could be interpreted to suggest that dabigatran may be especially useful in patients who cannot be well managed on warfarin.

The results of the three studies differed in terms of efficacy, safety, and tolerability. Dabigatran was found to be superior to warfarin in preventing stroke and systemic embolism at the highest dose studied (150 mg twice daily, dose approved for use in the USA) and non-inferior to warfarin for the safety endpoint [2]. Rivaroxaban was non-inferior to warfarin in both efficacy and safety, and apixaban was superior to warfarin in both efficacy and safety $[3,4]$. Of note, superior efficacy with apixaban was driven by the fact that fewer patients developed hemorrhagic stroke with the new agent, as compared to warfarin. Ischemic stroke rate was similar between warfarin and apixaban. Therefore, the primary benefit of apixaban may be that it is similar in effectiveness, but causes less important bleeding compared to warfarin. Gastrointestinal bleeding occurred more commonly with dabigatran and rivaroxaban than with warfarin [2]. Conversely, in the ARISTOTOLE study, gastrointestinal bleeding rate was similar between apixaban and warfarin. All three studies found a relative reduction of $33-59 \%$ in the rate of intracranial hemorrhage with the new antithrombotic agents compared to warfarin. Poor penetration of the blood-brain barrier in the case of dabigatran, and efflux of rivaroxaban and apixaban out of the brain by p-glycoprotein efflux pumps, have been proposed as potential explanations for these findings [8]. Generally, the new antithrombotic agents had few adverse events unrelated to bleeding. However, dabigatran was associated with a significantly higher rate of dyspepsia $(11.3 \%)$ compared to warfarin $(5.8 \%, p<0.001)$ in RE-LY
[2]. The higher rate of dyspepsia in RE-LY has been attributed to the acidic component contained within the dabigatran capsules [2]. The frequency of this adverse event may be a consideration when selecting an antithrombotic agent for a patient with gastroesophageal reflux disease.

\section{Organ dysfunction}

Pharmacokinetic differences exist between the three oral antithrombotic agents, particularly related to renal clearance and half-life. Dabigatran is largely eliminated through renal filtration. The half-life of dabigatran is approximately 13 hours when creatinine clearance is greater than $80 \mathrm{~mL} /$ minute and is close to 30 hours when creatinine clearance is below $30 \mathrm{~mL} /$ minute [9]. There is a 6-fold increase in drug exposure over time (area under the curve) with severe (creatinine clearance less than $30 \mathrm{~mL} / \mathrm{min}$ ute) renal dysfunction. Therefore, it is recommended to reduce the dose of dabigatran when creatinine clearance is less than 30 $\mathrm{mL} /$ minute and dabigatran is contraindicated when creatinine clearance is less than $15 \mathrm{~mL} /$ minute [9]. It should be noted that the reduced dose of dabigatran has not been studied in any clinical outcome study, but instead was derived from pharmacokinetic simulations that estimated that a therapeutic level of drug would be achieved when the lower dose was used in a patient with reduced kidney function [9].

In contrast to dabigatran, rivaroxaban was studied at both a full dose and an adjusted 'renal' dose. Approximately 25\% of patients in ROCKET-AF received the reduced dose of rivaroxaban. Therefore, there is more clinical experience with this dose in patients being treated for atrial fibrillation. It is also important to note that both rivaroxaban and apixaban are not as dependent on glomerular filtration rate for clearance because other mechanisms such as renal secretion and hepatic metabolism also play a role in the elimination of the drug $[10,11]$. Rivaroxaban and apixaban are not recommended for patients with moderate-to-severe liver disease due to lack of safety data, the role of hepatic metabolism in drug elimination, and the potential impact of hepatic failure on coagulation $[10,11]$.

Dabigatran appears to be the only agent of the three that is dialyzable. In a single-dose pharmacokinetic study of dabigatran $(50 \mathrm{mg}), 62 \%$ of the dose was removed after 2 hours of dialysis and $68 \%$ was removed after 4 hours [12]. Two published case reports also seem to indicate that hemodialysis may be useful for removal of dabigatran in patients with bleeding [13,14]. Data are not available evaluating the role of hemodialysis for removal of rivaroxaban and apixaban; however, neither is expected to be significantly dialyzable due to high protein binding with both agents $[10,11]$.

\section{Drug-drug interactions}

Dabigatran, rivaroxaban, and apixaban are all substrates of the p-glycoprotein (PGP) system. Rivaroxaban and apixaban are substrates of the cytochrome P-450 (CYP450) 3A4 system as well. Therefore, the drug-drug interaction potential for rivaroxaban and apixaban is greater than for dabigatran. Rifampin should not be used with any of the new antithrombotic agents because it is a strong inducer of PGP. The only other major clinically significant drug-drug interactions for dabigatran occur when ketoconazole 
or dronedarone (PGP inhibitors) are used in the setting of decreased kidney function (creatinine clearance of less than $50 \mathrm{~mL} /$ minute). When creatinine clearance is between 30 and $50 \mathrm{~mL} /$ minute the dose of dabigatran should be reduced by half, when used in combination with either ketoconazole or dronedarone. When creatinine clearance is less than $30 \mathrm{~mL} /$ minute, dabigatran should be avoided in combination with ketoconazole or dronedarone [9].

Rivaroxaban and apixaban have similar drug-drug interaction profiles. Since both agents are substrates of the PGP and CYP 450 3A4 systems, both agents interact with drugs that strongly inhibit or induce both of these systems concomitantly. However, labeling is slightly different for these two drugs. Rivaroxaban should be avoided with strong inhibitors such as ketoconazole, itraconazole, ritonavir, and conivaptan. Conversely, apixaban can be used with concomitant PGP and CYP450 3A4 agents, but the dose must be reduced to $2.5 \mathrm{mg}$ twice daily (if the patient is otherwise eligible to use the 5-mg twice-daily dose if no drugdrug interaction is present). If the patient is already taking the $2.5 \mathrm{mg}$ twice-daily dose due to increased serum creatinine, low body weight, and older age, then apixaban should be avoided with agents that concomitantly inhibit PGP and CYP450 3A4. Use of rivaroxaban or apixaban with strong inducers such as carbamazepine, phenytoin or rifampin should also be avoided.

\section{Remaining questions}

Some important issues remain unclear with regards to the use of any of the new antithrombotic agents. The first unresolved issue relates to the utility of laboratory monitoring of the new agents. Quantifying blood concentration or measuring the pharmacodynamic effects of the new antithrombotic agents is challenging with common laboratory tests available to clinicians today. The activated partial thromboplastin time (aPTT), prothrombin time (PT), international normalized ratio (INR), and thrombin time (TT) are generally increased when dabigatran, rivaroxaban or apixaban have been taken by the patient, but these tests are unable to be used to predict how much drug has been taken because there is not a predictable linear relationship between laboratory value and drug concentration, or because values are greater than the upper limit of detection at therapeutic doses [15]. Some newer tests, such as the ecarin clotting time (ECT) and dilute TT, seem to be useful for quantifying the antithrombotic effect of dabigatran, and chromogenic factor Xa assays could be useful for quantifying the effect of rivaroxaban and apixaban [15]. Unfortunately, these new tests are often unavailable or take an unreasonable amount of time to return results due to the need for off-site analysis. Even if laboratory monitoring does become readily available to quantify the effect of dabigatran, rivaroxaban, and apixaban, the type of patient who would benefit from monitoring would need to be more clearly defined.

Another outstanding question is whether or not the effects of the new antithrombotic agents can be reversed and, if so, what the optimal approach for reversal would be. There have been no clinical trials evaluating reversal approaches for dabigatran, rivaroxaban and apixaban. Animal studies and very limited experimental human studies have evaluated the role of concentrated blood factor products such as recombinant factor VIIa, prothrombin complex concentrates (PCC), and activated prothrombin complex concentrates (aPCC) on 'reversing' the antithrombotic effects of dabigatran and rivaroxaban [16-21]. Apixaban has not been extensively studied in this area, but it may be reasonable to assume that data with rivaroxaban could potentially be extrapolated to apixaban, given the similar mechanism of action of the two drugs [22]. Animal data with dabigatran and rivaroxaban are conflicting; however, aPCC seems to have the most consistent effect on the antithrombotic potential of these drugs $[18,19]$. The human study by Marlu and colleagues reported positive findings with aPCC as well, although this was an ex vivo study and as such interpretation is limited [21]. Recombinant factor VIIa does not seem to have much impact on bleeding with dabigatran, based on a published case report [13].

A major risk to consider with the attempted reversal of any antithrombotic with a concentrated blood factor product is thrombosis. Another complication of attempting reversal of a new oral antithrombotic agent is the lack of ability to rapidly monitor the patient in order to determine whether reversal attempts have been successful. Given that a clinician would only wish to reverse one of these antithrombotic agents in an emergent or life-threatening situation (major bleeding or need for urgent surgery/procedure), it may be warranted to attempt reversal with a concentrated blood factor product if the clinician feels that the thrombotic risk of these products is low for a given patient.

A final unresolved issue is whether these new agents will be cost-effective for patients with atrial fibrillation. The acquisition cost of these agents is high relative to the cost of warfarin. However, there are potential cost advantages of all three new antithrombotics. Specifically, one would expect the cost of monitoring the new agents to be less than warfarin, which requires routine INR monitoring and the time of a healthcare professional to manage the warfarin dosing. In addition, both dabigatran and apixaban demonstrated superiority for the efficacy endpoint of stroke and systemic embolism in RE-LY and ARISTOTLE, respectively. Improved patient outcomes would likely translate to reduced cost in the long run, especially given the debilitating nature of stroke and the impact of stroke on long-term healthcare needs. Reductions in major bleeding with apixaban and reductions in intracranial hemorrhage with all three agents, relative to warfarin, may also impact overall cost. Economic model studies have been published to support the cost-effectiveness of all three antithrombotic agents when compared to traditional clinicianmanaged warfarin therapy [23-25]. However, cost-effectiveness of the new agents in comparison to patient self-monitoring and self-management of warfarin has not been rigorously studied to date.

Due to the higher acquisition cost of the new agents relative to warfarin, clinicians need to ensure that the patient or the patient's medical insurance will pay for these medications. Nonadherence to drug therapy regimens can occur for a variety of reasons, and inability to pay for medications is a major reason. Non-adherence with the new antithrombotic agents has the potential to go undetected since there is no regular monitoring, as is carried out with warfarin. Healthcare professionals should be aware that if a patient is unable to pay for his/her therapy for 
stroke prevention, clinicians will in many cases only be able to ascertain this by discussing adherence with the patient and/or review of objective measures of adherence such as prescription refill history and timeliness or pill counts during clinic visits. Due to the shorter half-lives of the newer agents, missing one or two doses could theoretically put the patient at risk for an embolic event. Therefore, formal programs designed to monitor patient adherence may be necessary to achieve the results observed in the clinical trials of these medications.

\section{Agent selection considerations}

As the above review highlights, several similarities and differences should be considered when selecting from today's antithrombotic options in managing a patient with atrial fibrillation. At this point, it is unknown whether any of the three agents is more effective than another, because comparative effectiveness studies are unavailable. Therefore, patient safety will be one of the key criteria for selecting an antithrombotic strategy. Selecting the safest option for the patient requires assessment of kidney function. Any of the agents would be appropriate if creatinine clearance is greater than $50 \mathrm{~mL} /$ minute. Because the clinical trials of the new agents excluded patients with significant renal dysfunction (creatinine clearance less than $30 \mathrm{~mL} /$ minute for RE-LY and ROCKET-AF, and less than $25 \mathrm{~mL} /$ minute for ARISTOTLE), it may be prudent to avoid all of the new agents, in favor of warfarin, if creatinine clearance is less than $30 \mathrm{~mL} /$ minute. A conservative approach may be to avoid dabigatran in patients with a creatinine clearance between 15 and $30 \mathrm{~mL} /$ minute, because clinical data are not available to support the safety and effectiveness of this dose (despite dosing recommendations in the US product information [9]). It may also be prudent to avoid the use of rivaroxaban when creatinine clearance is between 15 and $30 \mathrm{~mL} / \mathrm{min}$ ute. While the reduced dose of rivaroxaban was studied in the ROCKET-AF study, patients with a creatinine clearance in this range were excluded [3]. If creatinine clearance is between 30 and $49 \mathrm{~mL} /$ minute, one may wish to consider either reduced-dose rivaroxaban, because clinical data support this dosing, or apixaban, because dose-adjustment for renal dysfunction is not necessary.

Another consideration when selecting an antithrombotic strategy is whether drug-drug interactions are present. Failure to identify relevant drug-drug interactions could lead to bleeding in the case of use of a new agent with inhibitors of metabolism (e.g., ketoconazole) or thrombosis if a new agent is used with an inducer of metabolism (e.g. rifampin). While warfarin may also interact with inhibitors or inducers of metabolism, the warfarin dose can be adjusted based on INR monitoring and would therefore be the safer choice. It may also be possible to use a reduced dose of apixaban in the setting of concomitant dual PGP and CYP3A4 administration and if the patient is eligible for the 5-mg twice-daily dose.

Other patient-specific considerations may also influence antithrombotic choice. Patients with pre-existing dyspepsia or gastroesophageal reflux disease may benefit from avoiding dabigatran. It may also be reasonable to avoid dabigatran and rivaroxaban in those with a history of gastrointestinal bleeding. Patients with limited financial means, or who have high insurance co-pay, may be better candidates for warfarin due to lower acquisition cost. Patients who may benefit from more frequent healthcare professional contact may also be better candidates for warfarin.

\section{Summary}

There are now more available choices for stroke prophylaxis in patients with atrial fibrillation than ever before. These new agents have advantages over warfarin, but bring new therapeutic dilemmas as well. Consideration of similarities and critical differences, including the effect of organ dysfunction on drug disposition, drug-drug interaction potential, and other patient-centered factors is essential for selecting the best antithrombotic therapy for the patient. It is also important to understand the impact of unanswered questions about these agents on the individual patient when deciding to use a new agent instead of warfarin.

\section{References}

1. Connolly SJ, Eikelboom J, Joyner C et al. Apixaban in patients with atrial fibrillation. N Engl J Med 2011;364:806-17.

2. Connolly SJ, Ezekowitz MD, Yusuf $S$ et al. Dabigatran versus warfarin in patients with atrial fibrillation. N Engl J Med 2009;361:1139-51.

3. Patel MR, Mahaffey KW, Garg J et al. Rivaroxaban versus warfarin in nonvalvular atrial fibrillation. N Engl J Med 2011;365:883-91.

4. Granger CB, Alexander JH, McMurray JJ et al. Apixaban versus warfarin in patients with atrial fibrillation. N Engl J Med 2011;365:981-92.

5. Schulman S, Kearon C; Subcommittee on Control of Anticoagulation of the Scientific and Standardization Committee of the International Society on Thrombosis and Haemostasis. Definition of major bleeding in clinical investigations of antihemostatic medicinal products in non-surgical patients. J Thromb Haemost 2005;3:692-4.

6. Gage BF, Waterman AD, Shannon W, Boechler M, Rich MW, Radford MJ. Validation of clinical classification schemes for predicting stroke: results from the National Registry of Atrial Fibrillation. JAMA 2001;285:2864-70.

7. Wallentin L, Yusuf S, Ezekowitz MD et al. Efficacy and safety of dabigatran compared with warfarin at different levels of international normalized ratio control for stroke prevention in atrial fibrillation: an analysis of the RE-LY trial. Lancet 2010;376:975-83.

8. San TR, Chan MYY, Siong TW et al. Stroke prevention in atrial fibrillation: understanding the new oral anticoagulants dabigatran, rivaroxaban, and apixaban. Thrombosis 2012, Article ID 108983, 10 pages. doi:10.1155/2012/108983.

9. Pradaxa (dabigatran) prescribing information. Ridgefield, CT: Boehringer Ingelheim Pharmaceuticals, Inc. December 2012. Available at: http://bidocs.boehringer-ingelheim.com/BIWebAccess/ViewServlet.ser?docBase $=$ renetnt $\&$ folderPath $=/$ Prescribing\%20Information/PIs/Pradaxa/Pradaxa.pdf [Last accessed: 24 February 2013].

10. Xarelto (rivaroxaban) prescribing information. Titusville, NJ: Janssen Pharmaceuticals, Inc. 2011. Available at: 
http://www.xareltohcp.com/sites/default/files/pdf/xarelto_0.pdf\#zoom=100 [Last accessed: 24 February 2013].

11. Eliquis (apixaban) prescribing information. Princeton, NJ: Bristol-Myers Squibb Company. December 2012. Available at: http://packageinserts.bms.com/pi/pi_eliquis.pdf [Last accessed: 24 February 2013].

12. Stangier J, Rathgen K, Stähle H et al. Influence of renal impairment on the pharmacokinetics and pharmacodynamics of oral dabigatran etexilate: an open-label, parallel-group, single-center study. Clin Pharmacokinet 2010;49:259-68.

13. Warkentin TE, Margetts P, Connolly SJ, Lamy A, Ricci C, Eikelboom JW. Recombinant factor VIIa (rFVIIa) and hemodialysis to manage massive dabigatran-associated postcardiac surgery bleeding. Blood 2012;119:2172-4.

14. Harinstein LM, Morgan JW, Russo N. Treatment of dabigatran-associated bleeding: case report and review of the literature. J Pharm Pract 2012 Nov 16 [Epub ahead of print].

15. Miyares MA, Davis K. Newer oral anticoagulants: a review of laboratory monitoring options and reversal agents in the hemorrhagic patient. Am J Health Syst Pharm 2012;69:1473-84.

16. Zhou W, Schwarting S, Illanes S et al. Hemostatic therapy in experimental intracerebral hemorrhage associated with the direct thrombin inhibitor dabigatran. Stroke 2011;42: 3594-9.

17. Godier A, Miclot A, Le Bonniec B et al. Evaluation of prothrombin complex concentrate and recombinant activated factor VII to reverse rivaroxaban in a rabbit model. Anesthesiology 2012;116:94-102.

18. van Ryn J, Ruehl D, Priepke H, Havel N, Wienen W. Reversibility of the anticoagulant effect of high doses of the direct thrombin inhibitor dabigatran, by recombinant factor VIIa or activated prothrombin complex concentrate. Haematologica. 2008; 93(s1):148. Abstract 0370. Available at: http://online.haematologica.org/eha13/browserecord. php?-action=browse $\&$-recid $=1923$ [Last accessed: 4 January 2013].

19. Gruber A, Marzek UM, Buetehorn U, Hanson S, Perzborn E. Potential of activated prothrombin complex concentrate and activated factor VII to reverse the anticoagulant effects of rivaroxaban in primates. Blood 2008;112 Abstract 3825. Available at: http://abstracts.hematologylibrary.org/cgi/content/abstract $/ 112 / 11 / 3825$ ? maxtoshow $=\&$ hits $=10 \&$ RESU LTFORMAT $=\&$ fulltext $=$ gruber $\&$ searchid $=1 \&$ FIRSTIN DEX $=0$ \&volume $=112$ \&issue $=11$ \&resourcetype $=$ HWCIT [Last accessed: 4 January 2013].

20. Eerenberg ES, Kamphuisen PW, Sijpkens MK, Meijers JC, Buller HR, Levi M. Reversal of rivaroxaban and dabigatran by prothrombin complex concentrate: a randomized, placebo-controlled, crossover study in healthy subjects. Circulation 2011;124:1573-9.

21. Marlu R, Hodaj E, Paris A, Albaladejo P, Cracowski JL, Pernod G. Effect of non-specific reversal agents on anticoagulant activity of dabigatran and rivaroxaban: a randomised crossover ex vivo study in healthy volunteers. Thromb Haemost 2012;108:217-24.

22. Blombäck M, He S, Bark N, Wallen HN, Elg M. Effects on fibrin network porosity of anticoagulants with different modes of action and reversal by activated coagulation factor concentrate. Br J Haematol 2001;152:758-65.

23. Freeman JV, Zhu RP, Owens DK et al. Cost-effectiveness of dabigatran compared with warfarin for stroke prevention in atrial fibrillation. Ann Intern Med 2011;154:1-11.

24. Lee S, Anglade MW, Pham D, Pisacane R, Kluger J, Coleman CI. Cost-effectiveness of rivaroxaban compared to warfarin for stroke prevention in atrial fibrillation. Am J Cardiol 2012;110:845-51.

25. Kamel H, Easton JD, Johnston SC, Kim AS. Cost-effectiveness of apixaban vs warfarin for secondary stroke prevention in atrial fibrillation. Neurology 2012;79:1428-34. 